\title{
AN EXAMPLE OF ALMOST FLAT AFFINE CONNECTIONS ON THE THREE-DIMENSIONAL SPHERE
}

\author{
YOSHIO AGAOKA \\ (Communicated by Christopher Croke) \\ Dedicated to Professor Masaru Takeuchi on his 60th birthday
}

\begin{abstract}
We show the existence of almost flat affine structures on the threedimensional sphere, and prove that the Pontryagin numbers serve as the obstruction to the existence of such structures.
\end{abstract}

In affine geometry, it is a natural and fundamental question whether a given manifold $M$ admits a torsion free flat affine connection or not. Concerning this problem, it is classically well known that the $n$-dimensional sphere $S^{n}$ does not admit such a connection for $n \geq 2$ because the fundamental group of $S^{n}$ is not infinite. (See [1].) In this note, we show that in the three-dimensional case, this non-existence is a subtle result, i.e., there exists an almost flat torsion free affine connection on $S^{3}$. To state the precise result, we first introduce the following concept.

Definition. A Riemannian manifold $(M, g)$ is called almost affinely flat if for any positive real number $\varepsilon$, there exists a torsion free affine connection $\nabla$ such that the norm of the curvature of $\nabla$ satisfies $\|R\|<\varepsilon$ at every point of $M$, where the norm $\|R\|$ is determined by the metric $g$. (Explicitly, $\|R\|=$ $\left\{1 / 2 \cdot \sum_{i j k l}\left(R_{k i j}^{l}\right)^{2}\right\}^{1 / 2}$, where $R\left(X_{i}, X_{j}\right) X_{k}=\sum_{l} R_{k i j}^{l} X_{l}$, and $\left\{X_{1}, \ldots, X_{n}\right\}$ is an orthonormal basis of $T_{x} M$.)

Note that, on a compact manifold, the notion does not depend on the metric, because the norms of tensor fields with respect to different metrics are within constants of each other. Now, our main result is stated as follows.

Theorem. The three-dimensional sphere $S^{3}$ is almost affinely flat.

Proof. To prove this theorem, we explicitly construct a family of connections $\nabla$ depending on a real parameter $t \in \mathbf{R} \backslash\{0\}$ such that the norm of the curvature of $\nabla$ with respect to the standard metric goes to 0 as $t \rightarrow 0$. Actually, by regarding $S^{3}$ as a Lie group, we can find such connections in the left-invariant category.

Received by the editors August 25, 1993 and, in revised form, May 31, 1994.

1991 Mathematics Subject Classification. Primary 53B05, 53C30; Secondary 57R20.

Key words and phrases. Affine connection, curvature, almost flat manifold, Pontryagin number. 
Let $\left\{X_{1}, X_{2}, X_{3}\right\}$ be an orthonormal left-invariant vector field on $S^{3}$ such that the bracket operation is given by

$$
\left[X_{1}, X_{2}\right]=2 X_{3}, \quad\left[X_{2}, X_{3}\right]=2 X_{1}, \quad\left[X_{3}, X_{1}\right]=2 X_{2} .
$$

Using these vector fields, we define a left-invariant torsion free affine connection by

$$
\begin{array}{lll}
\nabla_{X_{1}} X_{1}=t \cdot X_{1}, & \nabla_{X_{2}} X_{1}=t \cdot X_{2}, & \nabla_{X_{3} X_{1}}=t \cdot X_{3}, \\
\nabla_{X_{1}} X_{2}=t \cdot X_{2}+2 X_{3}, & \nabla_{X_{2}} X_{2}=-4 / t \cdot X_{1}, & \nabla_{X_{3} X_{2}}=-X_{1}, \\
\nabla_{X_{1}} X_{3}=-2 X_{2}+t \cdot X_{3}, & \nabla_{X_{2}} X_{3}=X_{1}, & \nabla_{X_{3}} X_{3}=-4 / t \cdot X_{1} .
\end{array}
$$

(This connection is actually $\mathrm{SO}(2)$-right invariant, i.e., it satisfies the additional equalities

$$
\left[X_{1}, \nabla_{X_{i}} X_{j}\right]=\nabla_{\left[X_{1}, X_{i}\right]} X_{j}+\nabla_{X_{i}}\left[X_{1}, X_{j}\right]
$$

for $i, j=1,2,3$.) Then by an easy calculation, we know that the curvature of this connection is given by

$$
R_{223}^{2}=R_{323}^{3}=-3 t \quad \text { and other } R_{k i j}^{l}=0 .
$$

For example, the $1 / t$-terms drop out by computations like

$$
\begin{aligned}
R\left(X_{1}, X_{2}\right) X_{2} & =\nabla_{X_{1}} \nabla_{X_{2}} X_{2}-\nabla_{X_{2}} \nabla_{X_{1}} X_{2}-2 \nabla_{X_{3}} X_{2} \\
& =\nabla_{X_{1}}\left(-4 / t \cdot X_{1}\right)-\nabla_{X_{2}}\left(t \cdot X_{2}+2 X_{3}\right)+2 X_{1} \\
& =-4 / t \cdot\left(t \cdot X_{1}\right)-t\left(-4 / t \cdot X_{1}\right)-2 X_{1}+2 X_{1} \\
& =0 .
\end{aligned}
$$

Therefore, $\|R\|^{2}=1 / 2 \cdot \sum_{i j k l}\left(R_{k i j}^{l}\right)^{2}=18 t^{2} \rightarrow 0$ as $t \rightarrow 0$, which proves the theorem.

Remark. The norm of the curvature of the bi-invariant affine connection on $S^{3}$ given by $\nabla_{X_{i}} X_{j}=1 / 2 \cdot\left[X_{i}, X_{j}\right]$ is equal to $\sqrt{6}$, and hence this canonical connection does not give the minimum of the function $\|R\|$ in the set of all left-invariant torsion free affine connections. But, by direct calculations, we can show that this connection gives the local minimum of $\|R\|$.

We do not know whether the result in this theorem holds for general $S^{n}$ $(n=2, n \geq 4)$. But not all compact manifolds are almost affinely flat. In fact, as we show in the next proposition, the Pontryagin numbers serve as the obstruction to the existence of such connections.

Proposition. Let $M^{4 n}$ be an almost affinely flat $4 n$-dimensional compact oriented manifold. Then the Pontryagin numbers of $M^{4 n}$ all vanish.

Proof. Let $L(M) \rightarrow M$ be the frame bundle of $M$ with structure group GL $(4 n, \mathbf{R})$, and let $\omega$ be the connection 1-form on $L(M)$ corresponding to $\nabla$. Then, by the Chern-Weil theory, each Pontryagin number $p$ is expressed as $p=\int_{M} f(\Omega)$, where $\Omega$ is the curvature form of $\omega$ and $f$ is an invariant polynomial of $\mathrm{GL}(4 n, \mathbf{R})$ with degree $2 n$ (cf. [4; II], [6]). Then, by using the pointwise inequality $\left|R_{k i j}^{l}\right| \leq\|R\|<\varepsilon$, we can easily prove

$$
|p|=\left|\int_{M} f(\Omega)\right|<\operatorname{const} \cdot \varepsilon^{2 n} \operatorname{vol}(M),
$$

and hence, the Pontryagin number $p$ must vanish. 
As an example, from this proposition, it follows that the complex projective space $P^{2 n}(\mathbf{C})$ is not almost affinely flat because the Pontryagin numbers of $P^{2 n}(\mathrm{C})$ are not zero (cf. [6]).

Remark. In [3], Gromov introduced the concept of almost flat ( $\varepsilon$-flat) manifolds in the category of Riemannian geometry. (See also [2].) In contrast to our case, the three-dimensional sphere $S^{3}$ (with any Riemannian metric) is not $\varepsilon$-flat in this sense for sufficiently small $\varepsilon$ because $S^{3}$ is not covered by a nilmanifold (cf. [2, p. 9]). We also remark that in the Riemannian case, the value of the curvature tensor $R_{k i j}^{l}$ of the Riemannian connection is unchanged even if we multiply the metric $g$ by a positive constant. In particular, by this modification, the value of the norm $\|R\|$ is unchanged for fixed \|\| .

In the special case where $M$ is a compact semi-simple Lie group, it is an interesting problem to determine the value $\inf \|R\|$ in the set of all left-invariant torsion free affine connections, where the norm \|\| is determined by a biinvariant metric. For example, in the case of $S^{3}$ or $\mathrm{SO}(3)$, we have clearly $\inf \|R\|=0$ from Theorem. In [5], it is proved that a semi-simple Lie group does not admit a torsion free left-invariant flat affine connection. But, this result does not imply inf $\|R\|>0$ as the above example shows.

\section{REFERENCES}

1. L. Auslander and L. Markus, Holonomy of flat affinely connected manifolds, Ann. of Math. (2) 62 (1955), 139-151.

2. P. Buser and H. Karcher, Gromov's almost flat manifolds, Astérisque 81 (1981).

3. M. Gromov, Almost flat manifolds, J. Differential Geom. 13 (1978), 231-241.

4. S. Kobayashi and K. Nomizu, Foundations of differential geometry. I, II, Wiley, New York, $1963,1969$.

5. H. Matsushima and K. Okamoto, Non-existence of torsion free flat connections on a real semisimple Lie group, Hiroshima Math. J. 9 (1979), 59-60.

6. J. W. Milnor and J. D. Stasheff, Characteristic classes, Ann. of Math. Stud., no. 76, Princeton Univ. Press, Princeton, NJ, 1974.

Department of Mathematics, Faculty of Integrated ARts \& Sciences, Hiroshima UniVERSITY, HIGASHIHIROSHIMA 739, JAPAN 